\title{
Thermo-Electric Energy Harvester for Low-Power Sanitary Applications
}

\author{
C. Beisteiner, B. G. Zagar \\ Institute for Measurement Technology, Johannes Kepler University, Linz, Austria, \\ christoph.beisteiner@jku.at, bernhard.zagar@jku.at
}

\begin{abstract}
:
The paper presents solutions for self-sufficient energy supply for sanitary applications. The difference in temperature between a hot and cold water pipe is utilized via a thermo-electric generator to feed a DC/DC converter. A sufficiently large voltage can be gained even for moderate temperature differences to power a DC/DC converter capable of stepping-up from low feed voltages in the range of a few millivolts. We could show that even the rather small temperature difference between ambient and cold water pipe temperatures is sufficient. An electronic circuit design is able to convert these voltages in the lower mV-range to necessary levels directly usable for low-power microprocessors and other electronic components. In particular these thermo-electric converters are used successfully for sanitary electronics even allowing to bridge typical idle times by lithium-ion cells. To achieve optimal energy utilization a theoretical model was devised that includes the thermo-electric converter the thermal capacity of the pipes and all thermal resistances involved. The modeling of the thermal section was done using the differential equations solver SPICE in order to allow calculatory efficiency and coupling with electrical loads or step-up converters.
\end{abstract}

Key words: energy harvesting, thermo-electric modelling, thermo-electric generator, heat exchanger

\section{Survey on Existing Possibilities for Energy- Harvesting Systems}

Tab. 1: Possible energy sources

\begin{tabular}{|c|c|c|}
\hline Source & Acceleration & Output power \\
\hline \multicolumn{3}{|c|}{ Mechanical - vibration/motion } \\
\hline $\begin{array}{l}\text { Vibrations of a } \\
\text { cargo container }\end{array}$ & $0.8 \mathrm{~m} / \mathrm{s}^{2} \mathrm{RMS}$ & $15 \mathrm{~mW}$ \\
\hline $\begin{array}{c}\text { Human / Closing } \\
\text { a door frame }\end{array}$ & $2.3 \mathrm{~m} / \mathrm{s}^{2}(120 \mathrm{~Hz})$ & $125-975 \mu \mathrm{W}$ \\
\hline Source & $\begin{array}{c}\text { Available } \\
\text { power density }\end{array}$ & $\begin{array}{c}\text { Electrical } \\
\text { power density }\end{array}$ \\
\hline \multicolumn{3}{|l|}{ Optical energy } \\
\hline Artificial light ${ }^{1}$ & $(40-150) \mu \mathrm{W} / \mathrm{cm}^{2}$ & $(4-15) \mu \mathrm{W} / \mathrm{cm}^{2}$ \\
\hline Sunlight $^{2}$ & $100 \mathrm{~mW} / \mathrm{cm}^{2}$ & $10 \mathrm{~mW} / \mathrm{cm}^{2}$ \\
\hline \multicolumn{3}{|l|}{ Thermal energy } \\
\hline Industrial & $100 \mathrm{~mW} / \mathrm{cm}^{2}$ & $(1-10) \mathrm{mW} / \mathrm{cm}^{2}$ \\
\hline Human $^{3}$ & $20 \mathrm{~mW} / \mathrm{cm}^{2}$ & $11 \mu \mathrm{W} /\left(\mathrm{cm}^{2} \mathrm{~K}\right)$ \\
\hline RF & $300 \mathrm{nW} / \mathrm{cm}^{2}$ & $100 \mathrm{nW} / \mathrm{cm}^{2}$ \\
\hline
\end{tabular}

Recent developments led to highly efficient lowpower electronics paving the way to their powering through ambient energy sources. The recent progress in the area of solar cell design, high density thermo-electric generators (TEG) electro-dynamic generators and piezoelectric transducers facilitates their use in energy-harvesting systems. A short survey is given in Table (1) on the power densities of various ambient energy resources [1],[2],[3],[4]. Several applications for all of these energy sources are described in [5].

To compare photovoltaic harvesters, direct sunlight and artificial light sources are considered. Various spectra and power densities attainable for different light sources were measured. The results are given in Table (2) for illuminance levels of $100 \mathrm{~lx}$.

Tab. 2: Optical power densities of current illuminations

\begin{tabular}{|c|c|}
\hline Illumination source & Typical power density \\
\hline $\begin{array}{l}\text { Incandescent light bulb } \\
\text { (Globo 230V/100W) }\end{array}$ & $135 \mu \mathrm{W} / \mathrm{cm}^{2}$ \\
\hline typical LED & $61 \mu \mathrm{W} / \mathrm{cm}^{2}$ \\
\hline $\begin{array}{l}\text { Fluorescent lamp (Osram } \\
\text { L36W/640) }\end{array}$ & $46 \mu \mathrm{W} / \mathrm{cm}^{2}$ \\
\hline
\end{tabular}

Ambient thermal power densities are found to be of similar magnitude, the useable power conversion efficiency, however, is subject to the thermodynamic Carnot efficiency. The efficien- 
cy of the TEGs depends strongly on the ratio of thermal, $\lambda$, vs. electrical conductivity, $\sigma$. TEGs typically use a semiconductor material (e.g. $\mathrm{Bi}_{2} \mathrm{Te}_{3}$ ), which combines low thermal conductivity, $\lambda$, with a still sufficient Seebeck coefficient, $\alpha$, and electrical conductivity $\sigma$. The power efficiency, $\eta$, at peak power and small temperature difference, $\Delta T$, thermal resistance $R_{\text {th }}$ and electrical resistance $R_{i}$ is given approximately by eq. (2) and no-load thermal voltage $\bigcup_{\text {th }}$ being:

$U_{t h}=\alpha \cdot \Delta T$

$\eta=\frac{P_{\text {out }}}{P_{\text {in }}}=\frac{1}{4} \alpha^{2} \Delta T \frac{R_{\text {th }}}{R_{i}}=\frac{1}{4} \alpha^{2} \Delta T \frac{\sigma}{\lambda}$

\section{Application in the Sanitary Field}

In the sanitary field nowadays mainly electronically controlled sanitary faucets spending water without the need for physical contact provide hygiene and reduce water consumption. Nevertheless, for optical sensor electronics and for switching the solenoid valve, sufficient power is needed. In order to detect a user interaction, a type of light barrier needs to operate intermittently the reflection off of the subjects hands needs to be detected via a down-stream amplifier circuit that needs to be powered, too. A simplified schematic is shown in Fig. 1. Modern processors facilitate a reduction of the current requirement to a range of $20 \mu \mathrm{A}$ on average by effective use of its sleep mode for this application. A highly power-efficient solenoid valve requires 200mA @ 6V but due to its bi-stable action an impulse duration of only $15 \mathrm{~ms}$ is sufficient. It is important to realize, however, that not only the active phase power requirement is met, but also long durations of idle mode interrupted only by short periods of scans for user interaction.

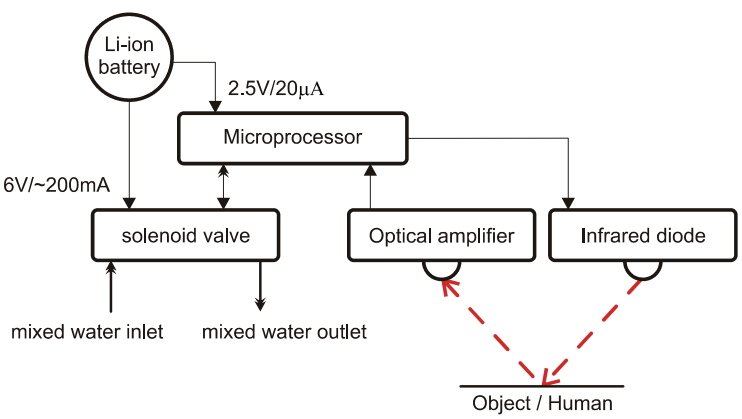

Fig. 1. Simple overview of sanitary electronics

\section{Sanitary Thermal Sources}

To convert thermal energy into electric energy, sufficient temperature differences are required which in our case are gained from the hot and cold water supply. Small reservoirs are included in the ducts to allow sustaining for several minutes (following each activation) the energy conversion to recharge the Li-ion battery. Fig. 2 shows a simplified heat flow diagram, the major temperature difference $\left(T_{\text {water,hot }}-T_{\text {water,cold }}\right)$ and thus the major no-load temperature drop is at the TEG ( $R_{T E G}$ its thermal resistance), thus maximizing its electric output voltage, if the heat resistances of the ducts $R_{\text {hot,side }}$ and $R_{\text {cold,side }}$ are negligible. This can be achieved by choosing thin-walled copper ducts. This, however, maintains to an increase of heat flowing from the warm to the cold side, which is not a problem in this case since the mixing temperature at the faucets will remain almost constant. Basically, inlet water temperatures are up to $60^{\circ} \mathrm{C}$ and cold water temperatures typically are at $12^{\circ} \mathrm{C}$ (they are required by law to be below $25^{\circ} \mathrm{C}$, risk of legionella if higher [6]). State of the art thermo-electric generators using multiple thermocouples in series exhibit thermal forces up to $220 \mathrm{mV} / \mathrm{K}$ (e.g.Thermalforce Inc.) with areas of $50 \times 50 \mathrm{~mm}^{2}$ and $140 \mathrm{mV} / \mathrm{K}$ (e.g. Micropelt Inc.) with $4.2 \times 3.4 \mathrm{~mm}^{2}$. Thus, already very low temperature differences result in considerable noload voltages.

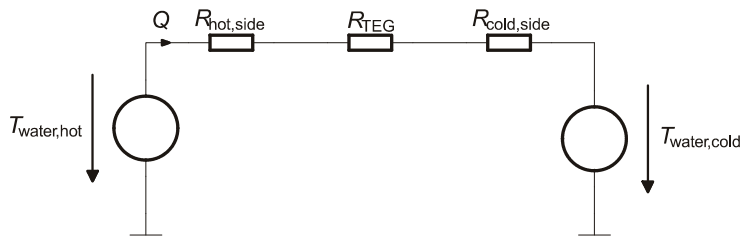

Fig. 2. Simple thermal equivalent circuit

Ultralow Input Voltage step-up Conversion

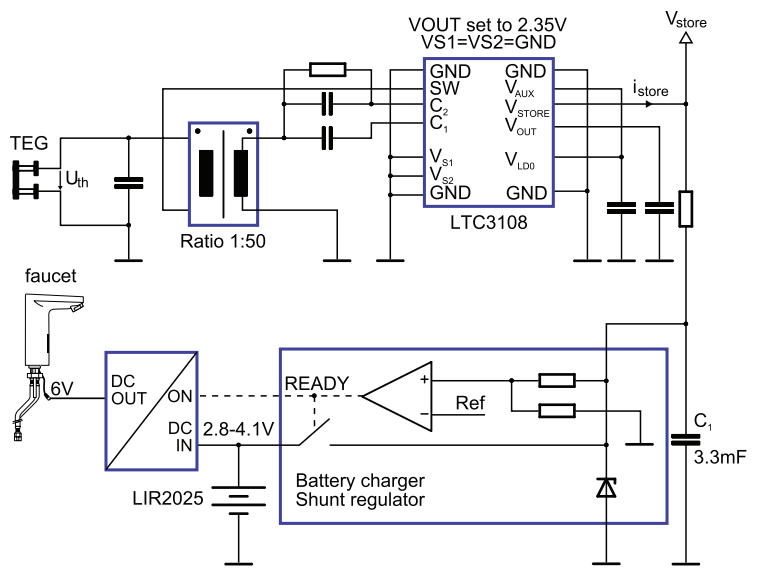

Fig. 3. Principle of the realized circuit

The company Linear-Technology Inc. produces an integrated circuit (LTC3108) that is able to operate from as low as $20 \mathrm{mV}$ generated upwards. Based on this integrated circuit, a PCB was designed to charge a Li-ion cell and to provide stable $6 \mathrm{~V}$ as output voltage. The Li-ion cell is buffering over periods, where no thermal input energy is available. As can been seen in 
Fig. 3 the Li-ion cell (LIR2025) is coupled to a battery charge controller preventing overcharge. In a further step, the voltage of the Liion cell is up converted again to $6 \mathrm{~V}$ in order to facilitate direct use by the sanitary faucet. The $\mathrm{DC} / \mathrm{DC}$ conversion to $6 \mathrm{~V}$ is done by a charge pump.

\section{Thermal Modelling}

In order to simulate the overall system a SPICE model was devised. All thermal components are converted to equivalent electric components. Most models are linear models, so an efficient SPICE simulation is possible. Furthermore LTC offers ready-made SPICE models for all of their components.

\section{Modeling the Thermo-Electric Generator}

In Fig. 4 an opened thermo-electric generator with $\mathrm{p}$ - and n-type semiconductor thermocouples is shown. Based on its physical principle a complete thermo- electric model

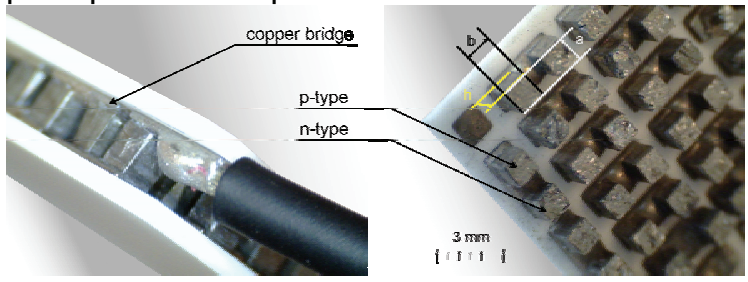

Fig. 4. Internal structure of a thermogenerator module; $p$ - and $n$ - semiconductors are connected via copper bridges. Typically a $\mathrm{Bi}_{2} \mathrm{Te}_{3}$-element, has an area of $1 \times 1 \mathrm{~mm}$ and a height of $3 \mathrm{~mm}$ including all coupling mechanisms is shown in Fig. 5. It was devised in order to optimize the thermal and the electrical output path.

The thermally generated voltage, $U_{\text {gen }}$, results from the electromotive force $\left(U_{\text {th }}=n \cdot \alpha \cdot \Delta T\right)$ diminished by ohmic losses $\left(n \cdot R_{i}, n\right.$ thermocouples connected electrically in series). The input impedance, $R_{L}$, of the $D C / D C$ converter is assumed stationary here (see eq. 3 ).

$n \alpha \Delta T=\left(n R_{i}+R_{L}\right) \cdot i_{\text {teg }}$

The thermal conductivity $\left(\mathrm{R}_{\mathrm{th}} / \mathrm{n}\right.$ of $\mathrm{n}$ thermocouples thermally parallel cause heat flow of $\left.\dot{Q}=\mathrm{n} \cdot \Delta \mathrm{T} / \mathrm{R}_{\mathrm{th}}\right)$, joule heating $\left(1 / 2 \cdot \mathrm{R}_{\mathrm{i}} \cdot \mathrm{i}_{\mathrm{teg}}{ }^{2}\right.$ split equally to both the hot and cold side) and a Peltier term $\left(n \cdot \alpha \cdot T_{\text {hot }} \cdot i_{\text {teg }}\right.$ and $n \cdot \alpha \cdot T_{\text {cold }} \cdot i_{\text {teg }}$ respectively) transporting heat from the hot to the cold side.

Heat $\dot{Q}_{\text {hot }}$ applied to the hot side (hot water) is

$$
\dot{Q}_{h o t}=n\left(\alpha T_{h o t} i_{t e g}-\frac{1}{2} R_{i} i_{t e g}{ }^{2}+\frac{\Delta T}{R_{t h}}\right)
$$

and heat dissipated at the cold side is

$$
\dot{Q}_{\text {cold }}=n\left(\alpha T_{\text {cold }} i_{\text {teg }}+\frac{1}{2} R_{i} i_{\text {teg }}{ }^{2}+\frac{\Delta T}{R_{\text {th }}}\right)
$$

Thermal resistances $R_{\text {hot }}$ and $R_{\text {cold }}$ summarize losses on the hot and cold side, respectively.

Fig. 5.

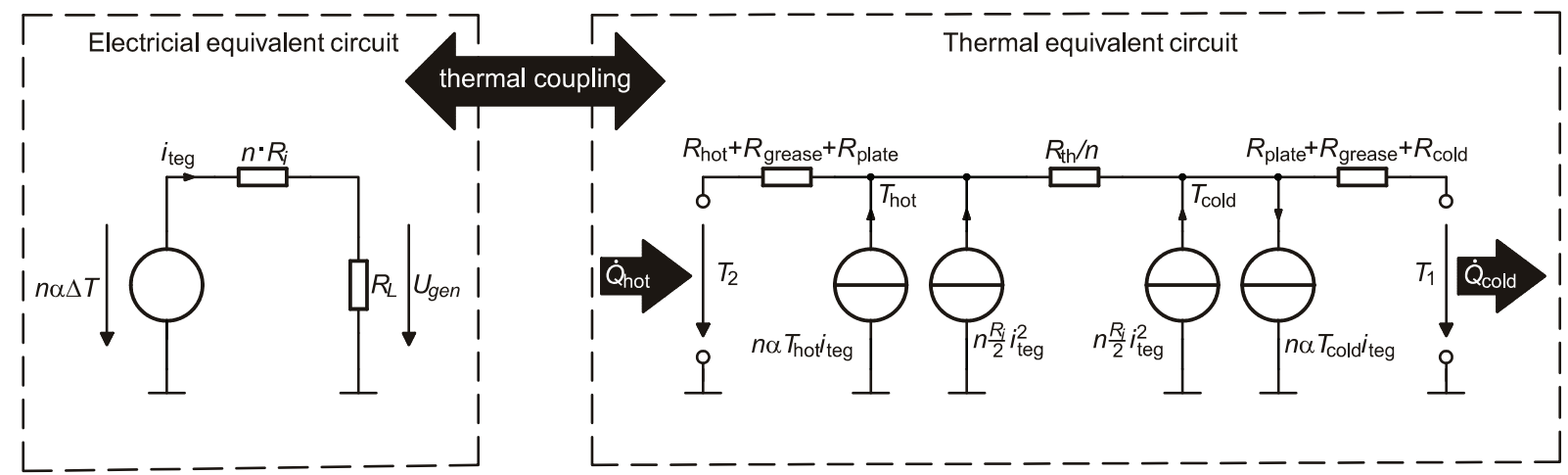

Stationary and coupled thermo-electric model in SPICE

The model parameters for a particular product of the manufacturer Thermalforce were identified. The number of thermocouples, $n / 2$, the open circuit voltage, $\mathrm{U}_{\mathrm{LL}}$, and a load situation with corresponding heat flow, $\dot{Q}_{h o t}$ and $\dot{Q}_{\text {cold }}$, and the load current, $i_{\text {load, }}$ without ceramic plates were taken from the data sheet. All temperatures are in Kelvin.

$$
\Delta T=T_{\text {hot }}-T_{\text {cold }}
$$

$$
\begin{aligned}
& R_{i}=\frac{i_{\text {load }} U_{L L}-\dot{Q}_{\text {hot }}+\dot{Q}_{\text {cold }}}{n \cdot i_{\text {load }}^{2}} \\
& R_{\text {th }}=\frac{2 n \Delta T^{2}}{\Delta T\left(\dot{Q}_{\text {hot }}+\dot{Q}_{\text {cold }}\right)-U_{L L} i_{\text {load }}\left(T_{\text {cold }}+T_{\text {hot }}\right)}
\end{aligned}
$$

$\alpha=\frac{U_{L L}}{n \cdot \Delta T}$ 
The thermal resistance, $R_{\text {plate, represents the }}$ effect of the ceramic plates. The small area of the thermocouples attached to the larger size plates has a heat spreading effect also modeled. The identification of $R_{\text {plate }}$ was done via equation (10), where $\dot{Q}_{h o t, p}, \dot{Q}_{\text {cold }, p}$ and $\mathrm{U}_{\mathrm{LL}, \mathrm{p}}$ are the values from the datasheet assuming heat flowing through the cross-section.

$$
R_{\text {plate }}=\frac{U_{L L} \cdot\left(T_{2}-T_{1}\right)-U_{L L, p} \cdot \Delta T}{U_{L L} \cdot\left(\dot{Q}_{\text {hot }, p}+\dot{Q}_{\text {cold }, p}\right)}
$$

Calculating these components for TEG 127$150-21$ leads to $R_{\text {plate }}=117 \mathrm{~K} / \mathrm{W}, \alpha=206 \mu \mathrm{V} / \mathrm{K}$ and $\mathrm{R}_{\mathrm{th}}=738 \mathrm{~K} / \mathrm{W}$.

\section{Mechanical Assembly}

The assembly consists of two heat exchangers one coupling the hot water to the TEG and the other to dissipate the heat from the cold side of the TEG to the cold water supply. They are made of copper to comply with design rules for drinking water supplies. Both contain reservoirs to increase their thermal capacity to typically power the DC/DC converter for approximately 30 minutes following an activation. The mounting was designed to reduce heat losses through bolts and other assembly parts. A conductive foil protects the TEG from mechanical stresses, forced by thermal expansion. The assembly process must take care of the brittleness of the TEG, which is circumvented by choosing a low module of elasticity of the conductive foil.

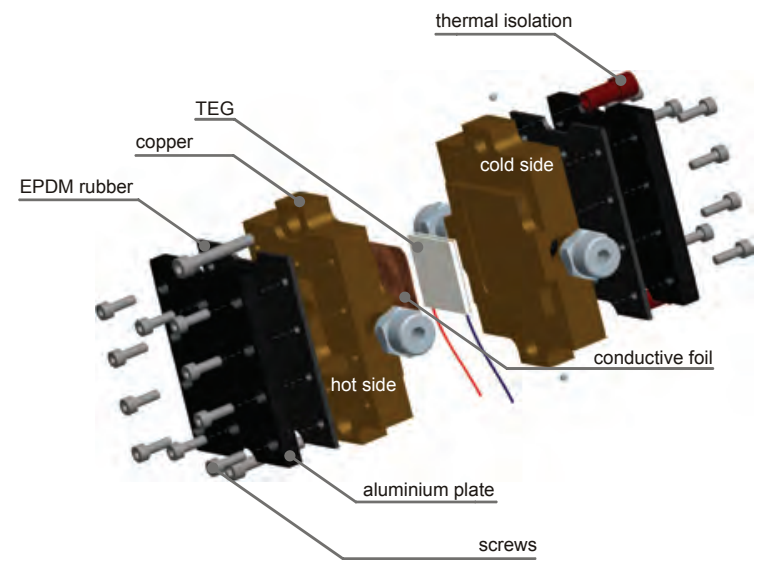

Fig. 6. Exploded assembly drawing of the energy harvester.

\section{Nonstationary Modelling}

For more precise simulations also heat capacitances need to be included. The main capacitance is concentrated in the copper mass and the water reservoirs. Due to the favourable thermal conductivity of copper, its thermal resistance can be disregarded. Modelling may be done in this case simply with a single thermal capacity. However, for the water block this condition is not fulfilled since water has a large thermal capacity and a low thermal conductivity. So a 2D-semi-discretization (see Fig. 7) of Fourier's thermal conduction equation with discrete heat capacities $C_{p}$

$$
C_{p}=\rho \cdot c_{p} \cdot \frac{l}{n_{z}} \frac{w}{n_{x}} t
$$

and discrete thermal resistances in $z$ - and $x$ direction was chosen [7].

$$
R_{z}=\frac{\frac{l}{n_{z}}}{\lambda_{\text {water }} \frac{w}{n_{x}} t}, R_{x}=\frac{\frac{w}{n_{x}}}{\lambda_{\text {water }} \frac{l}{n_{z}} t}
$$

The heat capacity $C_{p}$ is calculated with the specific heat capacity, $c_{p}$, the mass density, $\rho$, and the volume as determined by the length, $I$, width, $w$ and depth, $t$, of the cuboid water reservoir discretized with $n_{z}$ and $n_{x}$. Free convection for heat losses through air can be calculated with equations from the VDI Heat Atlas [8] and implemented with an electric current source in SPICE. Corresponding nonlinear material parameters can be stored in a look-up table. Similarly, the forced convection, caused by active flushings, can be implemented equally. During a flushing cycle, constant temperatures have to be forced on the discretized capacitors through SPICE switches. The result is that all heat capacities $C_{p}$ are charged with water of a specific temperature during a flushing cycle.

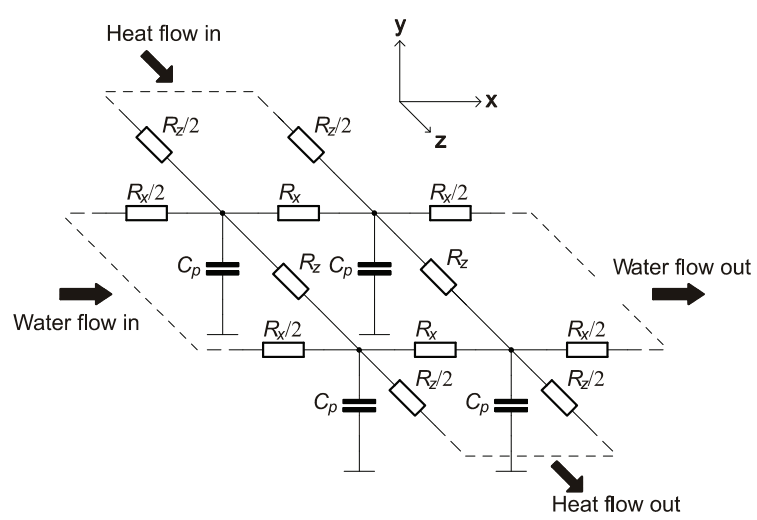

Fig. 7. $2 D$ heat transfer model.

\section{Modelling the LTC3108}

The transient simulation of the LTC3108 chip is possible, however, it is not adequate for thermal simulation due to the long simulation period. In the following an alternate electric model is presented, which can be used for stationary simulations. The simulation results are shown in Fig. 8. 

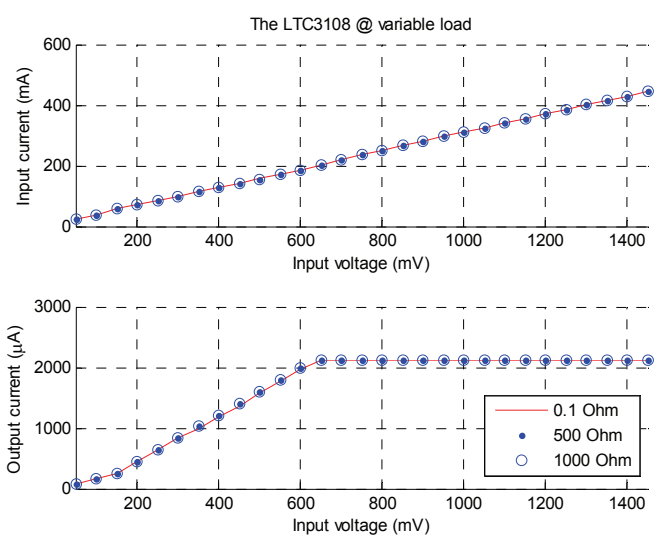

Fig. 8. Simulation results of the LTC3108 with a transformer ratio (see Fig. 3) of 1:50.

The input and output currents were simulated for the upper circuit in Fig. 3 including the store capacitor $\mathrm{C}_{1}$ and a resistive load between $0.1 \Omega$ and $1000 \Omega$. As can be seen, the input current is nearly independent of the load; the reason is to be found in the fact that the input device corresponds to an oscillator circuit with a downstream internal charge pump and a synchronized rectifier. The voltage of the synchronized rectifier, i.e. VAUX, is maintained at a constant voltage via a current limiter.

The output current is pulsed and limited to $2.13 \mathrm{~mA}$. It can also been seen, that the load current is nearly constant for small loads. The irregularities around the $0.15 \mathrm{~V}$ point cannot be explained without a more precisely known circuit set-up. However, they are small enough to be considered negligible. The result of the simulation is a very simple circuit diagram shown in Fig. 9 with parameters from Eq. (13) and (14).

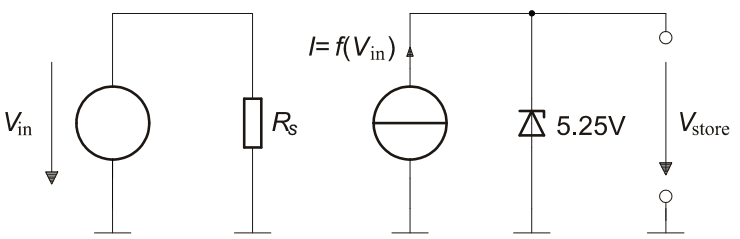

Fig. 9. Simplified stationary equivalent circuit for the ultralow voltage $D C / D C$ converter.

$R_{s}=3.2 \Omega$

$f(x)=\left\{\begin{array}{clc}0 \mathrm{~mA} & \text { for } & x<50 \mathrm{mV} \\ 4,47 \frac{\mathrm{mA}}{\mathrm{V}} \cdot x-324 \mu \mathrm{A} & \text { for } & \mathrm{x} \geq 50 \mathrm{mV} \text { and } \mathrm{x}<550 \mathrm{mV} \\ 2.13 \mathrm{~mA} & \text { for } & \mathrm{x} \geq 550 \mathrm{mV}\end{array}\right.$

\section{Experimental Results}

In order to verify the thermal model, temperature measurements were carried out on four surfaces (aluminium hot/cold, copper hot/cold) of the realized energy-harvester (Fig. 10) with a thermal camera. Due to the low thermal emission coefficient of galvanized (nickel-plated) surfaces, all areas to be measured were covered by an emission adhesive strip. One area was measured with a $\mathrm{CrNi}-\mathrm{Cr}$ thermocouple as reference. All temperature differences were exclusively measured with the thermal camera. Furthermore, the voltage at the thermo-electric generator was measured. The measurement and simulation results are shown in Fig. 11 and Fig. 12 and they agree very well.

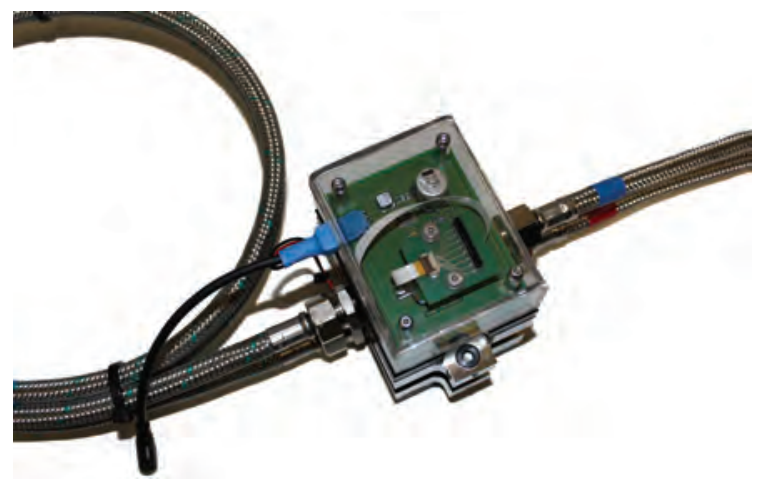

Fig. 10. The final energy harvester for sanitary applications.
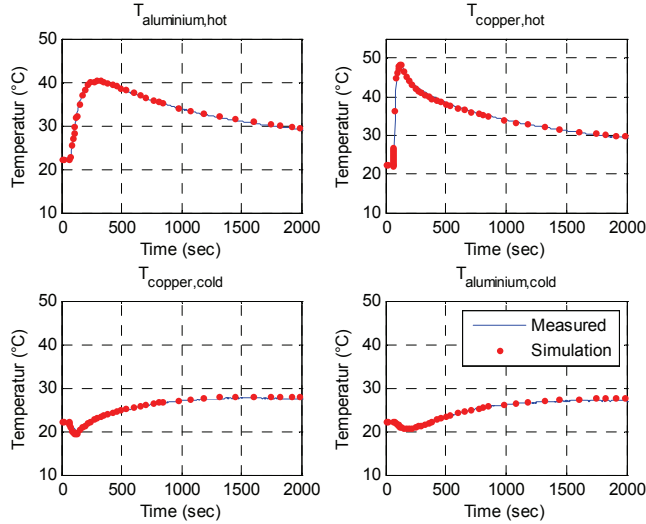

Fig. 11. Simulated and measured temperature curve at the aluminium and copper plates for excitation with $50^{\circ}$ hot and $18^{\circ}$ cold.

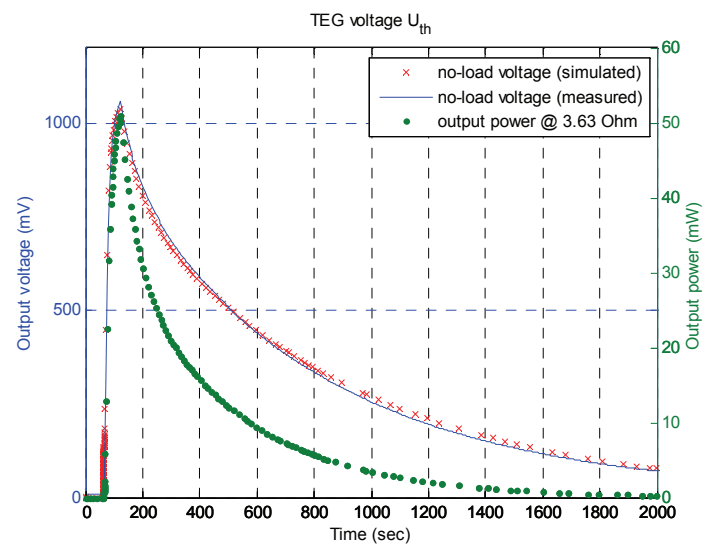

Fig. 12. Measurement, simulation and generated power compared. 


\section{Measurements on the Energy Harvester}

In order to show energy self-sufficiency, a measurement was done under real life conditions. As can been seen in Fig. 13 an average current of $1.5 \mathrm{~mA} @ 4.1 \mathrm{~V}$ is provided.
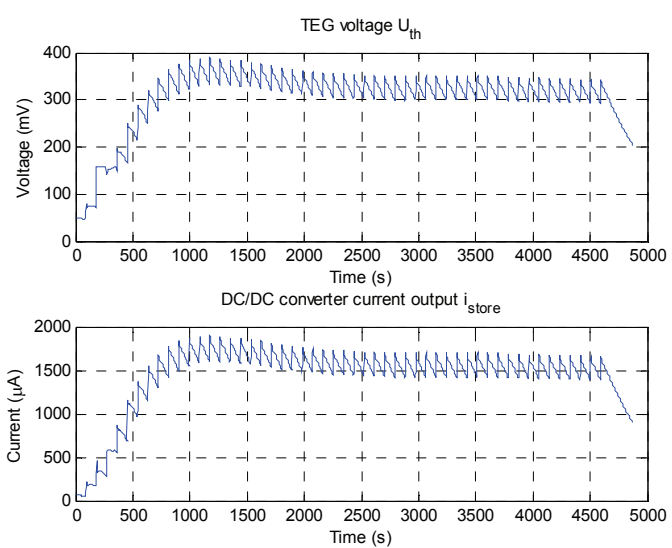

Fig. 13. Flushing every 90s with 3s water flow; which corresponds to approx. 1000 flushings per day

\section{Simulation Results}

From the above experimental and simulation results it can be concluded that the simulation is reliable.

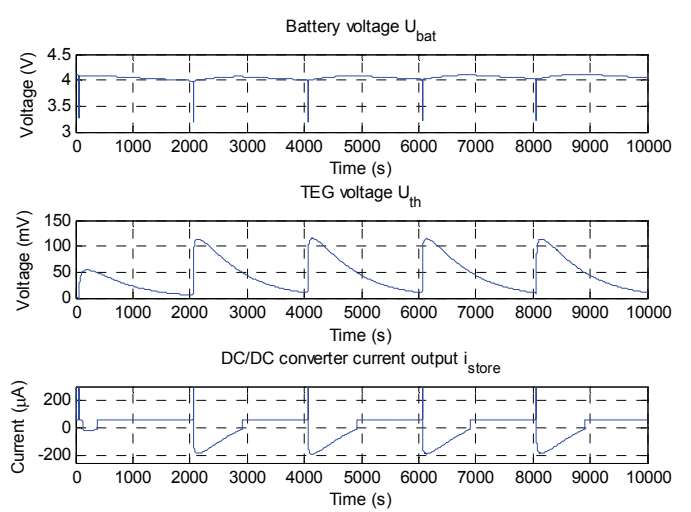

Fig. 14. Simulation with a flushing period of 2000s; approximately 50 flushings per day.

Thus we can simulate to characterize the system for very few daily cycles, which otherwise would be very time consuming in experimental testing. As can been shown in Fig. 15 the devise of system is capable of handling flushing cycles with intervals of 2000s. The charging process of the battery occurs only for approx. 800s. The peak currents occurring in Fig. 15 represent the current consumption of the solenoid valve during switching.

A more detailed analysis shows that an average output power up to $850 \mu \mathrm{W}$ could be reached with at least 100 flushing cycles per day.

\section{Conclusion}

A solution for a self-supplied sanitary mixing faucet has been presented. To this end, details in simulations and realization of the mechanics and electronics have also been shown. As the measurement and simulation results demonstrate, this energy harvester produces enough average power at different flushing cycles to guarantee self-sufficient energy sanitary mixing faucets.

\section{Acknowledgment}

The author gratefully acknowledges the partial financial support for the work presented in this paper by WIMTEC Elektronische Steuerungsund Meßgeräte $\mathrm{GmbH}$.

\section{References}

[1] S. Roundy, P. K. Wright, J. M. Rabaey, Energy scavenging for wireless sensor networks with special focus on vibrations, Kluwer Academic Publishers, 2004.

[2] Florian Maier, Michael Sturmlechner, Stefan Dierneder, Novel energy harvester with low friction losses, Systems, Signals and Devices, (2009); doi: 10.1109/SSD.2009.4956696

[3] R.J.M. Vullers, R. van. Schaijk, I. Doms, C. Van Hoof, R. Mertens, Micropower energy harvesting, Solid-State Electronics 53(7), 684-693 (2008); doi: 10.1016/j.sse.2008.12.011

[4] Shad Roundy, Energy Scavenging for Wireless Sensor Nodes with a Focus on Vibration to Electricity Conversion, dissertation in the University of California, Berkeley, Spring 2003

[5] Journal of Intelligent Material Systems and Structures Vol. 23, pp 1405-1542 (2012); ISSN: 1045-389X

[6] E DIN 1988-200, Trinkwasser-Installationen

[7] Nisagra N. Naik, Ashisha A. Ghangrekar, Sameer U. Kalghati, Simatha S. Sheety, Electrical and heat flow simulations of mems structures using spice, International Journal of Computational Engineering Science Vol. 4, No 2, 397-400 (2003); doi: 10.1142/S1465876303001368

[8] VDI Gesellschaft, VDI Heat Atlas, 2010 